\title{
Inverse national trends of laser interstitial thermal therapy and open surgical procedures for refractory epilepsy: a Nationwide Inpatient Sample-based propensity score matching analysis
}

\author{
Mayur Sharma, MD, MCh, Tyler Ball, MD, Ahmad Alhourani, MD, Beatrice Ugiliweneza, PhD, MSPH, \\ Dengzhi Wang, MS, Maxwell Boakye, MD, and Joseph S. Neimat, MD \\ Department of Neurosurgery, University of Louisville, Kentucky
}

OBJECTIVE Surgery for medically refractory epilepsy $(R E)$ is an underutilized treatment modality, despite its efficacy. Laser interstitial thermal therapy (LITT), which is minimally invasive, is increasingly being utilized for a variety of brain lesions and offers comparable seizure outcomes. The aim of this study was to report the national trends of open surgical procedures for RE with the advent of LITT.

METHODS Data were extracted using the ICD-9/10 codes from the Nationwide Inpatient Sample (NIS, 2012-2016) in this retrospective study. Patients with a primary diagnosis of RE who underwent either open surgeries (lobectomy, partial lobectomy, and amygdalohippocampectomy) or LITT were included. Patient demographics, complications, hospital length of stay (LOS), discharge disposition, and index hospitalization costs were analyzed. Propensity score matching (PSM) was used to analyze outcomes.

RESULTS A cohort of 128,525 in-hospital patients with RE was included and $5.5 \%(n=7045)$ of these patients underwent either open surgical procedures (94.3\%) or LITT (5.7\%). LITT is increasingly being performed at a rate of 1.09 per 1000 epilepsy admissions/year, while open surgical procedures are decreasing at a rate of $10.4 / 1000$ cases/year. The majority of procedures were elective (92\%) and were performed at large-bed-size hospitals (86\%). All LITT procedures were performed at teaching facilities and the majority were performed in the South (37\%) and West (30\%) regions. The median LOS was 1 day for the LITT cohort and 4 days for the open cohort. Index hospitalization charges were significantly lower following LITT compared to open procedures ( $\$ 108,332$ for LITT vs $\$ 124,012$ for open surgery, $p<0.0001$ ). LITT was associated with shorter median LOS, high likelihood of discharge home, and lower median index hospitalization charges compared to open procedures for RE on PSM analysis.

CONCLUSIONS LITT is increasingly being performed in favor of open surgical procedures. LITT is associated with a shorter LOS, a higher likelihood of being discharged home, and lower index hospitalization charges compared to open procedures. LITT is a safe treatment modality in carefully selected patients with RE and offers an opportunity to increase the utilization of surgical treatment in patients who may be opposed to open surgery or have contraindications that preclude open surgery. https://thejns.org/doi/abs/10.3171/2020.1.FOCUS19935

KEYWORDS national trends; outcomes; LITT; refractory epilepsy; Nationwide Inpatient Sample

$\mathrm{E}$ PILEPSY affects approximately 3.4 million people in the US, which is approximately $1.2 \%$ of the population. ${ }^{41}$ Despite advances in medical treatment, about $40 \%$ of cases are refractory to antiepileptic drugs and are referred to as medically refractory epilepsy (RE). ${ }^{10}$
The benefits of surgery for these patients have been well established by several studies, including a randomized controlled trial (RCT) in $2001 .{ }^{38}$ In that study, Wiebe et al. ${ }^{38}$ showed that $58 \%$ of patients with temporal lobe epilepsy (TLE) were without seizures impairing awareness

ABBREVIATIONS AHC = amygdalohippocampectomy; $A T L=$ anterior temporal lobectomy; IQR = interquartile range; LITT = laser interstitial thermal therapy; LOS = length of stay; $m$ TLE = mesial TLE; MTS = mesial temporal lobe sclerosis; NIS = Nationwide Inpatient Sample; PSM = propensity score matching; RCT = randomized controlled trial; RE = refractory epilepsy; SLAH = selective laser amygdalohippocampectomy; TLE = temporal lobe epilepsy.

SUBMITTED December 1, 2019. ACCEPTED January 27, 2020.

INCLUDE WHEN CITING DOI: 10.3171/2020.1.FOCUS19935. 
at 1 year following anterior temporal lobectomy (ATL), compared to $8 \%$ of patients who were randomized to the medical arm. The benefits of resection for RE were subsequently reinforced in two recent RCTs in $2012^{11}$ and 2017. ${ }^{8}$ However, despite this level 1 evidence, Englot et al., ${ }^{12}$ using the National (Nationwide) Inpatient Sample (NIS) database, reported that lobectomy for RE has decreased from $6.9 \%$ (1990-1994) to 4.3\% (2004-2008) of hospitalizations. The National Association of Epilepsy Centers annual surveys (2003-2012) reported that use of ATL for TLE decreased by more than 65\% between 2006 and 2012 and extratemporal lobe surgeries comprised $38 \%$ of all resective surgeries in 2012. ${ }^{17}$

Laser interstitial thermal therapy (LITT) is a minimally invasive surgical technique that has shown favorable outcomes in patients with a variety of brain lesions ${ }^{16,18,26 \text {, }}$ 27,32-34 and has shown favorable outcomes in patients with epilepsy. ${ }^{1,19,20,35,37,40}$ Following FDA clearance of two major LITT systems in 2007 (Visualase System, Medtronic Inc.) and 2009 (NeuroBlate System, Monteris Medical Corp.), this technology was first used to treat mesial TLE (mTLE) in 2010 and has been rapidly adapted as one of the neurosurgical tools to manage patients with RE. ${ }^{4,5,19,22,31,36,40} \mathrm{~A}$ recent multicenter study of 234 patients who underwent LITT for mTLE showed that $58 \%$ of patients achieved an Engel class I outcome at both 1 and 2 years following LITT. ${ }^{39}$

Given the increasing evidence of the efficacy of LITT, we looked at the trends of both open surgical treatment and LITT for RE using the NIS. We also aimed to identify the practice patterns and outcomes related to these procedures in patients with RE throughout the US. Our hypothesis was that LITT is being increasingly used in patients with RE, with a low incidence of complications and a higher likelihood to be discharged home compared to open procedures.

\section{Methods \\ Data Source}

Data from the NIS were used for this study, involving records from 2012 to 2016. The NIS was developed by the Agency for Healthcare Research and Quality for the Healthcare Cost and Utilization Project and is the largest available all-payer inpatient healthcare administrative data set in the US. ${ }^{15}$ It is compiled yearly and contains discharge data from more than 1000 hospitals located across 45 states. The NIS was designed to approximate a $20 \%$ stratified sample of all discharges from community hospitals, excluding rehabilitation and long-term acute care hospitals. These data enable researchers to make national estimates of healthcare utilization, access, charges, quality, and outcomes. We used the data from 2012 (year of full data following the introduction of LITT by the Centers for Medicare and Medicaid Services in 2011; https:// www.cms.gov/Regulations-and-Guidance/Guidance/ Transmittals/downloads/R2366CP.pdf) to 2016 (the last available data).

\section{Cohort Selection}

We queried the NIS data in a retrospective observation- al cohort design to extract patients with RE who underwent surgical treatment of intractable epilepsy using LITT or open surgery (lobectomy/partial lobectomy or amygdalohippocampectomy [AHC]), as described in previous studies. ${ }^{12,29}$ For this cohort, we evaluated in-hospital length of stay (LOS), total charges, and complications. The International Classification of Diseases, 9th (ICD-9) and 10th (ICD-10) Revision codes were used for extracting adult cases ( $\geq 18$ years of age) and are detailed in Supplementary Table 1 .

\section{Patient Characteristics}

Baseline characteristics were demographic variables (age, sex, race, insurance status, comorbidities, median household income for patient's zip code, and admission service type) and hospital characteristics (hospital region, bed size, and teaching status). Cases with missing variables were excluded. Comorbidities were measured with the Elixhauser comorbidity score, ${ }^{9}$ using the ICD-9-CM and ICD-10-CM code adaptations developed by Quan et al. ${ }^{28}$

\section{Outcomes of Interest}

The outcomes of interest were rates of LITT and open surgery per $1000 \mathrm{RE}$ admissions, hospital LOS, total charge, discharge disposition, complications, and mortality for each group. The charges were inflated to 2016 US dollars using the medical component of the Consumer Price Index (accessible through the US Bureau of Labor Statistics ${ }^{36}$ ). In-hospital complications were defined by the presence of at least one of the following complications: renal, nervous system, cerebrovascular disease, deep vein thrombosis or pulmonary embolism, pulmonary, infection, myocardial infarction, or pneumonia.

\section{Statistical Analysis}

To summarize continuous variables we used medians and interquartile ranges (IQRs), and for categorical variables we used using counts and percentages. Group comparisons were performed with linear regression analysis (for continuous variables) and Rao-Scott chi-square test (for categorical variables) specifically designed for sample survey data. Outcome comparisons were performed on propensity score-matched groups. The propensity score matching (PSM) technique consists of matching individuals in different groups with similar or close propensity scores. ${ }^{14}$ Patients who underwent LITT were matched 1 to 1 to patients who underwent open surgery. The propensity score was computed as the probability of undergoing an LITT using a multivariable logistic regression, in which all the patient characteristics were included as covariates. The matching process was performed using the greedy macro $^{3}$ with a caliper of 0.2 times the standard deviation of the propensity scores. Covariate balance before and after matching was evaluated using $p$ values. Trend analysis was performed by Joinpoint Trend Analysis software (downloaded in September 2019, version 4.7.0.0) from "Methods \& Tools for Population-based Cancer Statistics," Surveillance Research Program, National Cancer Institute. All other statistical data analysis was performed in SAS (version 9.4, SAS Institute, Inc.). 
TABLE 1. Characteristics and hospital demographics of patients extracted from the NIS database from 2012 to 2016

\begin{tabular}{|c|c|c|c|c|c|c|c|}
\hline \multirow[b]{2}{*}{ Variable } & \multirow{2}{*}{$\begin{array}{c}\text { Total RE } \\
(n=128,525)\end{array}$} & \multicolumn{3}{|c|}{ Nonmatched Cohort } & \multicolumn{3}{|c|}{ Propensity-Matched Cohort } \\
\hline & & $\operatorname{LITT}(n=400)$ & Open Op $(n=6645)$ & $p$ Value & LITT $(n=400)$ & Open Op $(n=400)$ & p Value \\
\hline \multicolumn{8}{|l|}{ Patient characteristics } \\
\hline Median age (IQR), yrs & $41(28-56)$ & $42(27-51)$ & $37(27-48)$ & 0.2186 & $42(27-51)$ & $36(27-50)$ & 0.5359 \\
\hline Females, $\mathrm{n}(\%)$ & $66,110(51)$ & $215(54)$ & $3355(50)$ & 0.5695 & $215(54)$ & $210(52)$ & 0.8591 \\
\hline Race, $n(\%)$ & & & & 0.0358 & & & 0.9119 \\
\hline White & $77,465(60)$ & $285(71)$ & $4540(68)$ & & $285(71)$ & $275(69)$ & \\
\hline Black & $21,420(17)$ & $15(4)$ & $525(8)$ & & $15(4)$ & $15(4)$ & \\
\hline Hispanic & $13,285(10)$ & $75(19)$ & $765(12)$ & & $75(19)$ & $90(22)$ & \\
\hline Others & $16,355(13)$ & $25(6)$ & $815(12)$ & & $25(6)$ & $20(5)$ & \\
\hline Insurance, n (\%) & & & & 0.3581 & & & 0.8189 \\
\hline Commercial & $38,560(30)$ & $180(45)$ & $3610(54)$ & & $180(45)$ & $155(39)$ & \\
\hline Medicaid & $34,135(27)$ & $100(25)$ & $1325(20)$ & & $100(25)$ & $110(28)$ & \\
\hline Medicare & $48,175(37)$ & $105(26)$ & $1415(21)$ & & $105(26)$ & $120(30)$ & \\
\hline Other & $7655(6)$ & $15(4)$ & $295(4)$ & & $15(4)$ & $15(4)$ & \\
\hline Elixhauser index, n (\%) & & & & 0.1188 & & & 0.4095 \\
\hline 1 & $34,170(27)$ & $180(45)$ & $2245(34)$ & & $180(45)$ & $210(53)$ & \\
\hline 2 & $32,135(25)$ & $105(26)$ & $2065(31)$ & & $105(26)$ & $105(26)$ & \\
\hline$\geq 3$ & $62,220(48)$ & $115(29)$ & $2335(35)$ & & $115(29)$ & $85(21)$ & \\
\hline $\begin{array}{l}\text { Median household income for } \\
\text { patient's zip code, } n(\%)\end{array}$ & & & & 0.0062 & & & 0.5759 \\
\hline Quartile 1 & $37,785(29)$ & $155(39)$ & $1480(22)$ & & $155(39)$ & $130(32)$ & \\
\hline Quartile 2 & $32,145(25)$ & $75(19)$ & $1805(27)$ & & $75(19)$ & $80(20)$ & \\
\hline Quartile 3 & $31,075(24)$ & $80(20)$ & $1680(25)$ & & $80(20)$ & $110(27)$ & \\
\hline Quartile 4 & $27,520(21)$ & $90(22)$ & $1680(25)$ & & $90(22)$ & $80(20)$ & \\
\hline Admission type, $\mathrm{n}(\%)$ & & & & 0.8417 & & & 0.717 \\
\hline Nonelective & $77,105(60)$ & $30(8)$ & $540(8)$ & & $30(8)$ & $25(6)$ & \\
\hline Elective & $51,420(40)$ & $370(92)$ & $6105(92)$ & & $370(92)$ & $375(94)$ & \\
\hline \multicolumn{8}{|l|}{ Hospital characteristics, n (\%) } \\
\hline Hospital region & & & & 0.0152 & & & 0.0593 \\
\hline Northeast & $27,390(21)$ & $75(19)$ & $1315(20)$ & & $75(19)$ & $30(8)$ & \\
\hline Midwest & $34,075(27)$ & $55(14)$ & $1865(28)$ & & $55(14)$ & $70(17)$ & \\
\hline South & $41,535(32)$ & $150(37)$ & $1995(30)$ & & $150(37)$ & $145(36)$ & \\
\hline West & $25,525(20)$ & $120(30)$ & $1470(22)$ & & $120(30)$ & $155(39)$ & \\
\hline Hospital bed size & & & & 0.0864 & & & $>0.99$ \\
\hline Small & $12,150(10)$ & $30(7.5)$ & $235(4)$ & & $30(7.5)$ & $30(8)$ & \\
\hline Medium & $27,195(21)$ & $30(7.5)$ & $690(10)$ & & $30(7.5)$ & $30(8)$ & \\
\hline Large & $89,180(69)$ & $340(85)$ & $5720(86)$ & & $340(85)$ & $340(85)$ & \\
\hline Hospital teaching status & & & & NA & & & NA \\
\hline Nonteaching & $22,485(17)$ & $0(0)$ & $110(2)$ & & $0(0)$ & $0(0)$ & \\
\hline Teaching & $106,040(83)$ & $400(100)$ & $6535(98)$ & & $400(100)$ & $400(100)$ & \\
\hline
\end{tabular}

$\mathrm{NA}=$ not applicable.

Boldface type indicates statistical significance.

\section{Results}

\section{Participants and National Trends}

Using the NIS database, we found a decreasing trend for open surgical procedures for RE: $10.9 \%$ of patients underwent open surgeries from 1998 to 2001, 11.1\% from 2002 to $2006,7.5 \%$ from 2007 to 2011, and 5.5\% from 2012 to 2016 (Fig. 1). We identified a cohort of 128,525 patients with RE from 2012 to 2016, 5.5\% of whom underwent surgical treatment (open or LITT surgeries). Of these patients with RE, 69\% were admitted at large hospitals and 83\% at teaching hospitals. Teaching hospitals were more likely to offer surgeries compared to nonteaching hospitals; Table 1). Open surgical procedures accounted for $94.3 \%$ of the surgical treatments, while LITT accounted for 5.7\% during the study period. There was an increasing utiliza- 


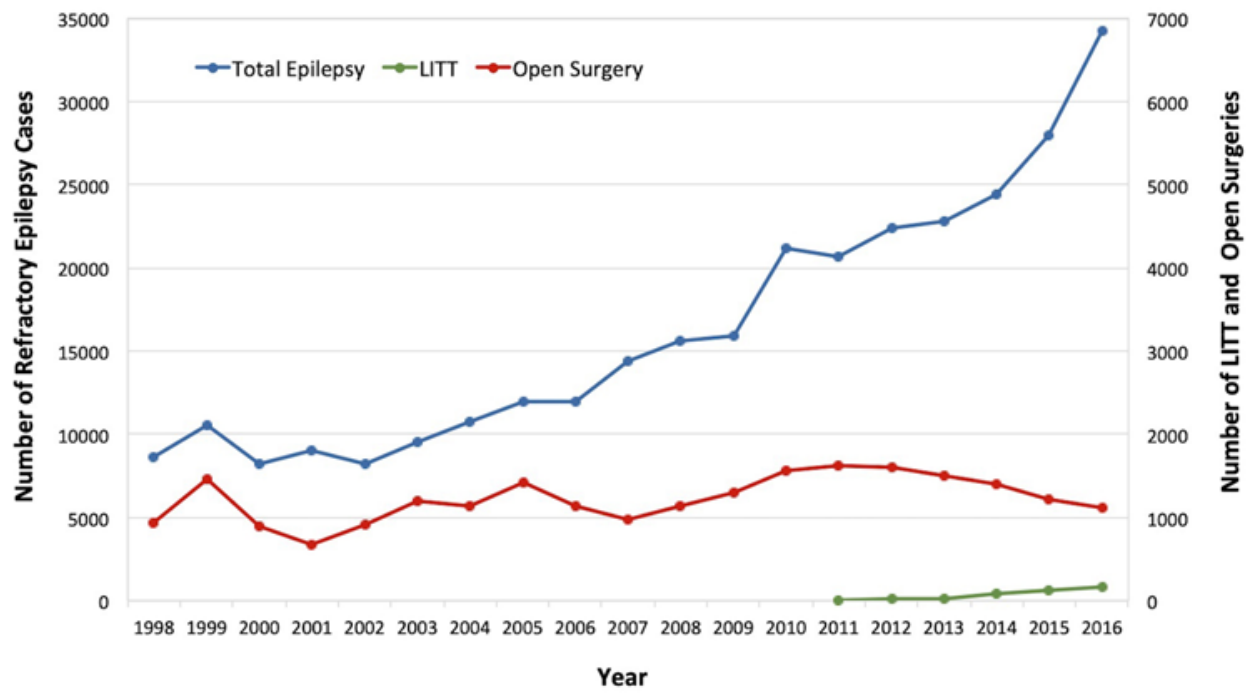

FIG. 1. Graph showing the national trends of total number of RE cases, open surgical procedures, and LITT for RE from 1998 to 2016.

tion of LITT and a decreasing utilization of open surgery during the study period. Overall, all surgical procedures (LITT and open) showed a downward trend. LITT procedures increased at a rate of 1.09 per $1000 \mathrm{RE}$ admissions/ year, while open surgical procedures decreased at a rate of 10.4 per $1000 \mathrm{RE}$ admissions/year from 2012 to 2016 (Figs. 2 and 3). LITT for RE has gradually increased from $1 \%$ in 2012 to $13.2 \%$ in 2016.

\section{Patient and Hospital Demographics}

Patient and hospital characteristics are summarized in Table 1 . The median patient age was 42 years old for the LITT cohort and 37 years old for the open surgery cohort. Female sex was evenly distributed and the majority of patients were white in both cohorts. Approximately half of the patients had commercial insurance (LITT 45\%, open surgery 54\%) and median household income in the 1st or 2nd quartiles (LITT 58\%, open surgery 50\%). One-third of the patients had an Elixhauser comorbidity index of 3+ (LITT 29\%, open surgery 35\%). The majority of procedures were elective ( $92 \%$ for both LITT and open surgery) and performed at high-volume hospitals. Interestingly, all LITT procedures were performed at teaching facilities and the majority of these procedures were performed in South $(37 \%)$ or West $(30 \%)$ region hospitals. We found that only $9 \%(n=35)$ of patients in the LITT cohort underwent either intracranial or video EEG during index hospitalization compared to $31 \%(n=2085)$ in the open cohort $(\mathrm{p}<0.0001)$.

\section{Outcome Data, Adverse Events, and Follow-Up}

Median hospital LOS was 1 day for the LITT cohort and 4 days for the open cohort ( $p<0.0001$ ). Only $4 \%$ of patients in the LITT cohort had postprocedure complications compared to $15 \%$ in the open cohort $(p=0.006)$. Cerebrovascular followed by neurological, pulmonary, and infectious complications were noted following either of these procedures. There were no deaths in the LITT cohort, whereas there was a $0.4 \%$ mortality rate in the open surgery cohort. Additionally, $96 \%$ of patients following LITT were discharged home compared to $86 \%$ of patients following open surgical procedures $(p=0.0017)$. Median index hospitalization charges were significantly lower following LITT compared to open procedures (LITT $\$ 108,332$, open $\$ 124,012$, p < 0.0001; Table 2, Fig. 4).

Following PSM, we found that LITT procedures were associated with shorter median LOS, high likelihood of discharge home, and lower median index hospitalization charges compared to open procedures for RE (Table 2).

\section{Discussion}

In our retrospective cohort study, we found a significant underutilization of surgical procedures for RE with an overall decrease in the rate of surgeries from 2012 to 2016, as described in the literature. ${ }^{12,13}$ LITT was performed as an elective procedure at high-volume centers at teaching facilities, whereas only $2 \%$ of open procedures were performed at nonteaching facilities. This is the first study highlighting the trends and comparing the clinical outcomes following LITT and open surgeries (lobectomy/ partial lobectomy/AHC) in patients with RE.

\section{National Trends of Open Procedures and LITT}

Englot et al., ${ }^{12}$ using the NIS database, reported that although there were increased hospitalizations for RE from 1990 to 2008, there was no corresponding increase in the numbers of lobectomies, resulting in an overall decrease in surgery rates. They reported that open procedures were performed in $6.9 \%$ of patients who underwent hospitalization for RE from 1990 to 1994, compared to 4.3\% of hospitalizations from 2004 to 2008..$^{12}$ In our study, we found that $7.5 \%$ of patients underwent open procedures for RE from 2007 to 2011, which decreased to 5.5\% from 2012 to 2016. Open surgical procedures started showing a down- 

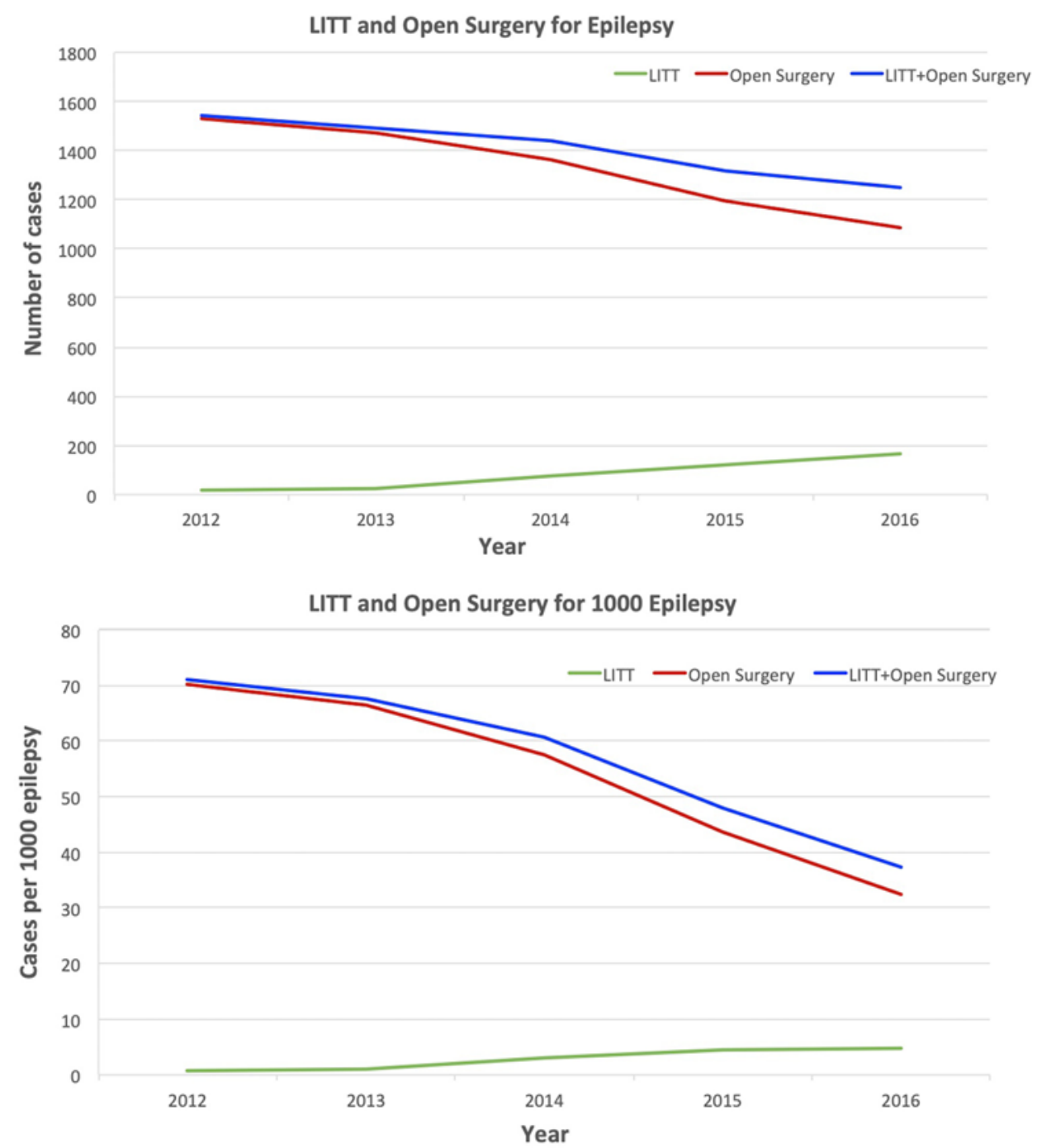

FIG. 2. Graph showing the national trends of LITT, open surgical procedures, and combined LITT and open procedures for RE total cases (upper) and per 1000 epilepsy admissions (lower).

ward trend beginning with the years 2011-2012 following the first report of LITT published by Curry et al. ${ }^{5}$ However, the rate of increase in LITT utilization does not match the decrease in open surgeries. There are many factors that may contribute to underutilization of the surgical treatment of RE, such as referral pattern to specialized epilepsy surgery centers, ${ }^{10,13}$ misconceptions related to surgery for RE, ${ }^{10}$ insurance status, healthcare access and referral to within-insurance networks, ${ }^{21}$ and utilization of neurostimulator techniques such as vagus nerve stimulation, ${ }^{17}$ deep brain stimulation, and responsive neurostimulation. ${ }^{2}$ However, future research is needed to further analyze the impact of neurostimulation procedures (which are primarily used for extratemporal and nonlesional epilepsies) on ablative procedures.

Englot et al. ${ }^{12}$ attributed this decrease to increased hospitalizations, primarily at low-volume hospitals, which were less likely to offer open surgical procedures. In their subsequent study, the authors reported an increasing trend of open procedures performed at middle-volume hos- pitals (5-15 procedures/year) and a decreasing trend at high-volume hospitals ( $>15$ procedures/year).$^{13}$ Similarly, we found that $69 \%$ of patients were admitted for RE at large-bed-size hospitals; however, $86 \%$ of open surgical procedures and $85 \%$ of LITT procedures were performed at these centers. The authors also reported that $6.5 \%$ of patients received lobectomies at teaching and 3.6\% at nonteaching hospitals for RE. ${ }^{12}$ This trend has shifted toward teaching hospitals from 2012 to 2016, as shown in the present study $(6.5 \%$ teaching and $0.5 \%$ nonteaching hospitals). This suggests that the rate of surgery at comprehensive epilepsy centers may be similar, but a decrease may be due to a decreased utilization of epilepsy surgery at nonteaching hospitals, as reported by Englot et al..$^{12}$ It is concerning that an increased referral shift to comprehensive epilepsy centers has not occurred given the American Academy of Neurology guidelines suggesting the importance of referral to such centers. This important topic is beyond the scope of this paper but certainly one that bears further investigation. 

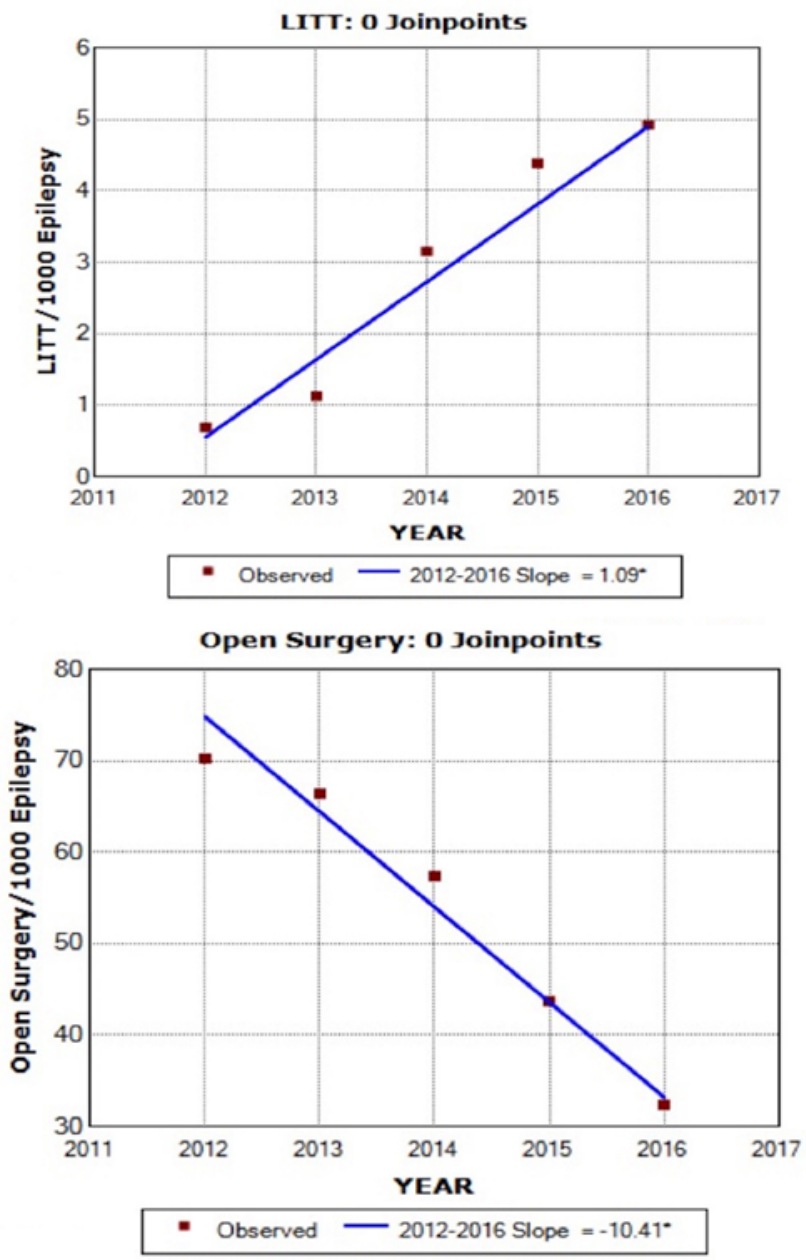

FIG. 3. Results from Joinpoint regression software showing the national trends of LITT (upper) and open procedures (lower) from 2012 to 2016 in the US. ${ }^{*}$ Indicates that the slope is significantly different from zero at the alpha $=0.05$ level.

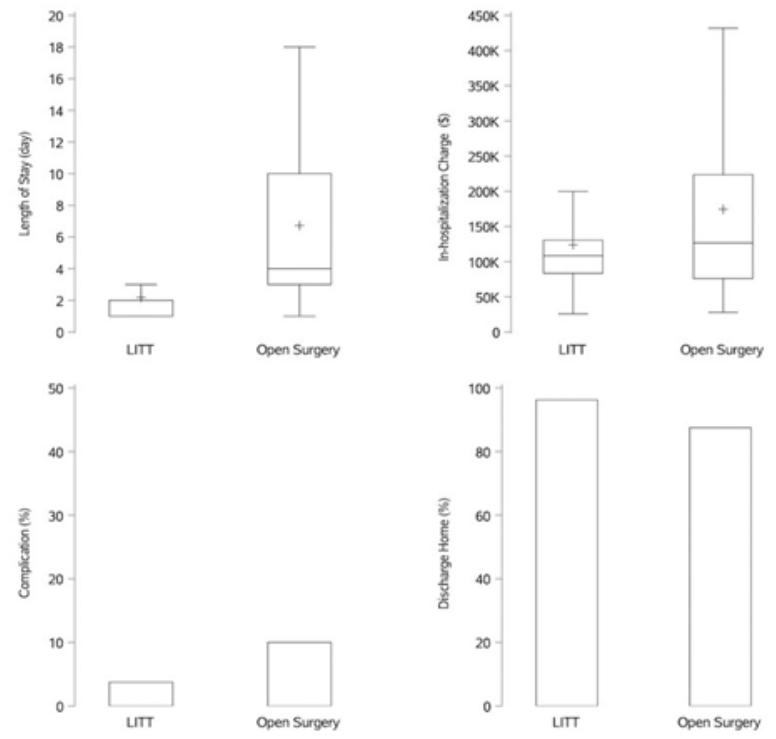

FIG. 4. Upper: Boxplots showing hospital LOS and index hospitalization charges in patients who underwent LITT and open surgical procedures for RE. Lower: Bar graph showing complications and discharges home following LITT and open surgical procedures for RE.

\section{LITT Versus Open Surgical Procedures for RE}

A recent multicenter retrospective study (11 centers and 234 patients) utilizing LITT for mTLE reported that 58\% of patients achieved Engel class I outcomes at both 1 and 2 years. ${ }^{39}$ Another retrospective study reported Engel class I seizure freedom in $56 \%(n=10 / 18)$ of patients with mesial temporal lobe sclerosis (MTS) and 58\% $(\mathrm{n}=7 / 12)$ of patients with non-MTS following selective laser amygdalohippocampectomy (SLAH) in patients with mTLE..$^{40}$ There were no differences in outcomes among groups with MTS and non-MTS in this and other studies. ${ }^{6,39,40}$ Other studies reported Engel class I seizure freedom rates of $36.4 \%$ at 1 year $(\mathrm{n}=20),{ }^{19} 52 \%$ at $24 \pm 11$ months $(\mathrm{n}=21),{ }^{35}$ and $62 \%$ at $18 \pm 12$ months $(\mathrm{n}=30)^{23}$ following SLAH for TLE. These results are comparable to the first RCT for TLE (n $=40$ in the surgical arm and $n=40$ in the medical arm), which reported that $58 \%$ of patients were without seizures

TABLE 2. Outcomes in patients who underwent LITT and open procedures for RE

\begin{tabular}{|c|c|c|c|c|c|c|}
\hline \multirow[b]{2}{*}{ Outcomes } & \multicolumn{3}{|c|}{ Nonmatched Cohort } & \multicolumn{3}{|c|}{ Propensity-Matched Cohort } \\
\hline & $\operatorname{LITT}(n=400)$ & Open Op $(n=6645)$ & p Value & $\operatorname{LITT}(n=400)$ & Open Op $(n=400)$ & $\mathrm{p}$ Value \\
\hline $\begin{array}{l}\text { Median hospital } \\
\text { LOS (IQR), } \\
\text { days }\end{array}$ & $1(1-1)$ & $4(2-9)$ & $<0.0001$ & $1(1-1)$ & $3(2-10)$ & $<0.0001$ \\
\hline $\begin{array}{l}\text { Median charge } \\
\text { (IQR), \$ }\end{array}$ & $108,332(83,236-129,865)$ & $124,012(73,651-219,409)$ & $<0.0001$ & $108,332(83,236-129,865)$ & $126,627(75,651-222,465)$ & 0.0029 \\
\hline $\begin{array}{l}\text { Complications, } \\
\mathrm{n}(\%)\end{array}$ & $15(4)$ & $980(15)$ & 0.0066 & $15(4)$ & $40(10)$ & 0.1105 \\
\hline $\begin{array}{l}\text { Discharge } \\
\text { home, } \mathrm{n}(\%)\end{array}$ & $385(96)$ & $5700(86)$ & 0.0017 & $385(96)$ & $350(88)$ & 0.0137 \\
\hline Mortality, n (\%) & $0(0)$ & $25(0.4)$ & NA & $0(0)$ & $<10$ & NA \\
\hline
\end{tabular}

Boldface type indicates statistical significance. 
impairing awareness and 38\% were free of any seizures at 1 year following temporal lobectomy. Schmeiser et al. ${ }^{30}$ and Dorfer et al. ${ }^{7}$ recently reported Engel IA outcome rates of $63.4 \%$ (total $n=134$ ) at 1 year and $78.4 \%$ (total $n=158$ ) at last follow-up, respectively, following transsylvian AHC in patients with mTLE. Based on these studies, LITT offers similar clinical outcomes in terms of seizure freedom compared to open surgical procedures. The NIS database is limited in extracting the clinical outcome data following LITT, and thus is a limitation of our study as mentioned below.

\section{LOS, Complications, and Mortality Following LITT and Open Surgeries}

The median hospital LOS was 1 day and $96 \%$ of patients were discharged home following LITT in our study; these results are in accordance with previously published studies. ${ }^{19,23,40}$ Following open surgical procedures, the median LOS was 4 days and $86 \%$ of patients were discharged home. Rumalla et al., ${ }^{29}$ utilizing a national readmission database, reported that the mean LOS was 8.3 days and $84 \%$ of patients had a favorable discharge disposition. This difference may be attributable to inclusion of "hemispherectomy" in open surgical procedures in this series, which may have skewed the results.

A recent multicenter study reported an overall complication rate of $15 \%$ in 234 patients following LITT for mTLE. ${ }^{39}$ A total of 42 complications in 35 patients were reported, but only 34 were permanent and 1 patient died in this series, which was attributed to sudden unexplained death in epilepsy. ${ }^{39}$ Youngerman et al. ${ }^{40}$ reported only transient complications (superior quadrantanopia, incomplete ablation, and progressive headache secondary to small intracerebral/intraventricular hemorrhage) in 3 of 30 patients, and no mortality was reported following LITT in this study. Similarly, Kang et al. ${ }^{19}$ reported serious complications in 1 patient (5\%) and minor complications (headache $[n=4]$, insomnia [ $n=1]$, scalp numbness $[n=1]$, and transient worsening of mood symptoms $[n=2])$ in 8 patients following LITT.

Wiebe et al. ${ }^{38}$ reported complications in $4(10 \%)$ of 40 patients following temporal lobectomy for TLE in the surgical arm. Dorfer et al. ${ }^{7}$ reported transient complications in 7 patients $(4.6 \%)$ with no permanent deficits or mortality in this series following open surgical procedures. However, 3 of these 7 patients underwent surgical management of the complications (hygroma $[n=2]$ and intracerebral hemorrhage $[n=1])$. Similarly, Schmeiser et al. ${ }^{30}$ reported postoperative complications in 30 patients (18.5\%), permanent complications in 4 patients $(2.5 \%)$, and no perioperative mortality following AHC. Martens et al. ${ }^{24}$ reported unexpectedly high rates of temporal infarctions (47.9\%) and frontal infarctions (10.4\%) following transsylvian $\mathrm{AHC}$ and temporal infarctions may have contributed to better seizure outcomes in these patients. Englot et al. ${ }^{13}$ in their NIS-based study (1990-2008) reported an increased incidence of perioperative complications at low-volume $(12.9 \%)$ and middle-volume $(10 \%)$ centers compared to high-volume (6.1\%) centers. Similarly, McClelland et al. ${ }^{25}$ reported an overall perioperative complication rate of $8 \%$ with no mortality following lobectomy for RE using the
NIS database (1988-2003). Interestingly, these authors did not find an impact for hospital case volume on postoperative morbidity; only increasing patient age and nonprivate insurance predicted increasing perioperative morbidity and adverse discharge disposition in that study. ${ }^{25}$ The authors stratified high caseload as $\geq 3$ lobectomies/year in this study ${ }^{25}$ compared to $>15$ lobectomies/year by Englot et al. ${ }^{13}$ In our study, we found that $15 \%$ of patients experienced complications following open procedures compared to $4 \%$ following LITT ( $\mathrm{p}<0.05$ ); however, on matched analysis no differences in complications were noted following either of these procedures.

\section{Index Hospitalization Charges}

A recent study reported mean total costs of $\$ 49,779 \pm$ $\$ 5125$ using a national readmission database in 1587 patients following open surgical procedures (hemispherectomy/lobectomy and partial lobectomy) for RE. ${ }^{29}$ Another recent study reported that payments associated with LITT for brain tumors were comparable to other treatment modalities (LITT \$89,785 $\pm \$ 15,885$, and other treatments $\$ 82,042 \pm \$ 22,070)$. The authors used national Medicare averages for surgery and reported costs related to physician services, postoperative radiation therapy, chemotherapy, rehabilitation, and palliative or hospice care. In our study, we found that median index hospitalization charges following LITT were significantly lower compared to open procedures (LITT $\$ 108,332$ vs open $\$ 124,012$, p $<0.05$ ). This difference may be attributed to utilization of different databases, inclusion of all insurance types (commercial, Medicare, Medicaid), and reporting hospital charges instead of costs in our study.

\section{Future Directions}

Although the surgical treatment for TLE is well established following 3 RCTs published in 2001, ${ }^{38} 2012,{ }^{11}$ and $2017,{ }^{8}$ there remains significant underutilization of open surgeries for RE. ${ }^{12,13}$ The findings of our study are concordant with these previously published trends. Since the introduction of LITT with its minimally invasive technology, similar outcome profile, and low complication rates with healthcare utilization (compared to open procedures), we believe that LITT offers an attractive option to both patients and physicians. With increasing trends of LITT utilization, this technology is likely to fill the gap and help patients who are either not medically fit or not willing to undergo open surgeries for RE in the future.

\section{Strengths and Limitations of the Study}

Our study provides an insight into the national trends and practice patterns of LITT and open procedures for RE throughout the US from 2012 to 2016 . The NIS also provides an opportunity to look at complications, index hospitalization charges, discharge dispositions, and mortality in a large cohort of patients.

Apart from inherent limitations of being a retrospective study, such as selection and treatment biases, coding error leading to misclassification bias is a unique limitation of administrative databases. The low sample size of the LITT cohort, and the NIS representing only $20 \%$ of 
hospital admissions, may limit generalizability of these results. Therefore, results need to be interpreted in light of these limitations. Furthermore, granular clinical and imaging data cannot be extrapolated from this database, which limits the understanding regarding the surgical decision-making process. Surgical trajectory, number of catheters/procedure, amount of tissue that needs to be ablated to achieve satisfactory seizure outcome, and patients requiring repeat procedures cannot be extrapolated from the NIS. Also, it is not feasible to differentiate the complexity of cases among the cohorts. Similarly, the exact localization of seizure focus and type of resection cannot be discerned using the NIS. Clinical follow-up and healthcare utilization cannot be extracted using the NIS. In our study, we found that only $9 \%(n=35)$ of patients in the LITT cohort underwent either intracranial or video EEG during index hospitalization compared to $31 \%(n=2085)$ in the open cohort. However, because it is not feasible to have a retrospective or follow-up period among the cohorts using the NIS database, we are likely to include only patients who underwent intracranial/video EEG along with LITT or open surgical procedures during the "index hospitalization." This may not truly reflect the clinical practice patterns, where patients are likely to undergo a preoperative workup with intracranial/video EEG, etc., prior to hospitalization for the definitive surgical procedure (LITT or open surgeries). Nevertheless, our study is the first one to highlight the increasing trends of LITT for RE and compare the outcomes between LITT and open procedures using the NIS database. Future prospective clinical trials are required to answer clinical questions that cannot be answered using the NIS.

\section{Conclusions}

Overall, surgery for RE (open and LITT) showed a downward trend during the study years. LITT is increasingly being performed, whereas the use of open procedures is declining. LITT is associated with a shorter LOS, a higher likelihood of being discharged home, and lower index hospitalization charges compared to open procedures. LITT is a safe treatment modality in carefully selected patients with RE and offers an opportunity to increase the utilization of surgical treatment in patients who may be opposed to open surgery or have contraindications that preclude open surgery.

\section{Acknowledgments}

We would like to thank Thomas Ryan Chandler, MPH, for assistance with data extraction.

\section{References}

1. Ball T, Sharma M, White AC, Neimat JS: Anterior corpus callosotomy using laser interstitial thermal therapy for refractory epilepsy. Stereotact Funct Neurosurg 96:406-411, 2018

2. Benbadis SR, Geller E, Ryvlin P, Schachter S, Wheless J, Doyle W, et al: Putting it all together: options for intractable epilepsy: an updated algorithm on the use of epilepsy surgery and neurostimulation. Epilepsy Behav 88S:33-38, 2018

3. Bergstralh EJ, Kosanke JL: Computerized matching of cases to controls. Technical report no. 56. Mayo Foundation, 1995 (http://www.mayo.edu/research/documents/biostat-56pdf/ doc-10026923) [Accessed February 14, 2020]

4. Bezchlibnyk YB, Willie JT, Gross RE: A neurosurgeon's view: laser interstitial thermal therapy of mesial temporal lobe structures. Epilepsy Res 142:135-139, 2018

5. Curry DJ, Gowda A, McNichols RJ, Wilfong AA: MR-guided stereotactic laser ablation of epileptogenic foci in children. Epilepsy Behav 24:408-414, 2012

6. Donos C, Breier J, Friedman E, Rollo P, Johnson J, Moss L, et al: Laser ablation for mesial temporal lobe epilepsy: surgical and cognitive outcomes with and without mesial temporal sclerosis. Epilepsia 59:1421-1432, 2018

7. Dorfer C, Czech T, Aull-Watschinger S, Baumgartner C, Jung R, Kasprian G, et al: Mesial temporal lobe epilepsy: long-term seizure outcome of patients primarily treated with transsylvian selective amygdalohippocampectomy. J Neurosurg 129:174-181, 2018

8. Dwivedi R, Ramanujam B, Chandra PS, Sapra S, Gulati S, Kalaivani M, et al: Surgery for drug-resistant epilepsy in children. N Engl J Med 377:1639-1647, 2017

9. Elixhauser A, Steiner C, Harris DR, Coffey RM: Comorbidity measures for use with administrative data. Med Care 36:8-27, 1998

10. Engel J Jr: The current place of epilepsy surgery. Curr Opin Neurol 31:192-197, 2018

11. Engel J Jr, McDermott MP, Wiebe S, Langfitt JT, Stern JM, Dewar S, et al: Early surgical therapy for drug-resistant temporal lobe epilepsy: a randomized trial. JAMA 307:922-930, 2012

12. Englot DJ, Ouyang D, Garcia PA, Barbaro NM, Chang EF: Epilepsy surgery trends in the United States, 1990-2008. Neurology 78:1200-1206, 2012

13. Englot DJ, Ouyang D, Wang DD, Rolston JD, Garcia PA, Chang EF: Relationship between hospital surgical volume, lobectomy rates, and adverse perioperative events at US epilepsy centers. J Neurosurg 118:169-174, 2013

14. Faries DE, Leon AC, Haro JM, Obenchain RL: Analysis of Observational Health Care Data Using SAS. Cary, NC: SAS Institute Inc, 2010

15. HCUP National Inpatient Sample (NIS): Healthcare Cost and Utilization Project (HCUP). Rockville, MD: Agency for Healthcare Research \& Quality, 2012 (https://hcup-us. ahrq.gov/nisoverview.jsp) [Accessed February 14, 2020]

16. Ivan ME, Mohammadi AM, De Deugd N, Reyes J, Rodriguez $\mathrm{G}$, Shah A, et al: Laser ablation of newly diagnosed malignant gliomas: a meta-analysis. Neurosurgery 79 (Suppl 1):S17-S23, 2016

17. Kaiboriboon K, Malkhachroum AM, Zrik A, Daif A, Schiltz NM, Labiner DM, et al: Epilepsy surgery in the United States: analysis of data from the National Association of Epilepsy Centers. Epilepsy Res 116:105-109, 2015

18. Kamath AA, Friedman DD, Hacker CD, Smyth MD, Limbrick DD Jr, Kim AH, et al: MRI-guided interstitial laser ablation for intracranial lesions: a large single-institution experience of 133 cases. Stereotact Funct Neurosurg 95:417-428, 2017

19. Kang JY, Wu C, Tracy J, Lorenzo M, Evans J, Nei M, et al: Laser interstitial thermal therapy for medically intractable mesial temporal lobe epilepsy. Epilepsia 57:325-334, 2016

20. Karsy M, Patel DM, Halvorson K, Mortimer V, Bollo RJ: Anterior two-thirds corpus callosotomy via stereotactic laser ablation. Neurosurg Focus 44 (VideoSuppl2):V2, 2018

21. Kreider AR, French B, Aysola J, Saloner B, Noonan KG, Rubin DM: Quality of health insurance coverage and access to care for children in low-income families. JAMA Pediatr 170:43-51, 2016

22. LaRiviere MJ, Gross RE: Stereotactic laser ablation for medically intractable epilepsy: the next generation of minimally invasive epilepsy surgery. Front Surg 3:64, 2016 
23. Le S, Ho AL, Fisher RS, Miller KJ, Henderson JM, Grant GA, et al: Laser interstitial thermal therapy (LITT): seizure outcomes for refractory mesial temporal lobe epilepsy. Epilepsy Behav 89:37-41, 2018

24. Martens T, Merkel M, Holst B, Brückner K, Lindenau M, Stodieck S, et al: Vascular events after transsylvian selective amygdalohippocampectomy and impact on epilepsy outcome. Epilepsia 55:763-769, 2014

25. McClelland S III, Guo H, Okuyemi KS: Population-based analysis of morbidity and mortality following surgery for intractable temporal lobe epilepsy in the United States. Arch Neurol 68:725-729, 2011

26. Mohammadi AM, Sharma M, Beaumont TL, Juarez KO, Kemeny H, Dechant C, et al: Upfront magnetic resonance imaging-guided stereotactic laser-ablation in newly diagnosed glioblastoma: a multicenter review of survival outcomes compared to a matched cohort of biopsy-only patients. Neurosurgery 85:762-772, 2019

27. Patel P, Patel NV, Danish SF: Intracranial MR-guided laserinduced thermal therapy: single-center experience with the Visualase thermal therapy system. J Neurosurg 125:853860, 2016

28. Quan H, Sundararajan V, Halfon P, Fong A, Burnand B, Luthi JC, et al: Coding algorithms for defining comorbidities in ICD-9-CM and ICD-10 administrative data. Med Care 43:1130-1139, 2005

29. Rumalla K, Smith KA, Arnold PM, Schwartz TH: Readmission following surgical resection for intractable epilepsy: nationwide rates, causes, predictors, and outcomes. Oper Neurosurg (Hagerstown) 16:374-382, 2019

30. Schmeiser B, Wagner K, Schulze-Bonhage A, Elger CE, Steinhoff BJ, Wendling AS, et al: Transsylvian selective amygdalohippocampectomy for mesiotemporal epilepsy: experience with 162 procedures. Neurosurgery 80:454-464, 2017

31. Schwarzmaier HJ, Yaroslavsky IV, Yaroslavsky AN, Fiedler V, Ulrich F, Kahn T: Treatment planning for MRI-guided laser-induced interstitial thermotherapy of brain tumors - the role of blood perfusion. J Magn Reson Imaging 8:121-127, 1998

32. Shah AH, Semonche A, Eichberg DG, Borowy V, Luther E, Sarkiss CA, et al: The role of laser interstitial thermal therapy in surgical neuro-oncology: series of 100 consecutive patients. Neurosurgery [epub ahead of print], 2019

33. Sharma M, Balasubramanian S, Silva D, Barnett GH, Mohammadi AM: Laser interstitial thermal therapy in the management of brain metastasis and radiation necrosis after radiosurgery: an overview. Expert Rev Neurother 16:223232, 2016

34. Silva D, Sharma M, Juthani R, Meola A, Barnett GH: Magnetic resonance thermometry and laser interstitial thermal therapy for brain tumors. Neurosurg Clin N Am 28:525533,2017
35. Tao JX, Wu S, Lacy M, Rose S, Issa NP, Yang CW, et al: Stereotactic EEG-guided laser interstitial thermal therapy for mesial temporal lobe epilepsy. J Neurol Neurosurg Psychiatry 89:542-548, 2018

36. United States Bureau of Labor Statistics. BLS.gov (https:// www.bls.gov/) [Accessed February 14, 2020]

37. Vakharia VN, Sparks R, Li K, O’Keeffe AG, Miserocchi A, McEvoy AW, et al: Automated trajectory planning for laser interstitial thermal therapy in mesial temporal lobe epilepsy. Epilepsia 59:814-824, 2018

38. Wiebe S, Blume WT, Girvin JP, Eliasziw M: A randomized, controlled trial of surgery for temporal-lobe epilepsy. $\mathbf{N}$ Engl J Med 345:311-318, 2001

39. Wu C, Jermakowicz WJ, Chakravorti S, Cajigas I, Sharan $\mathrm{AD}$, Jagid JR, et al: Effects of surgical targeting in laser interstitial thermal therapy for mesial temporal lobe epilepsy: a multicenter study of 234 patients. Epilepsia 60:1171-1183, 2019

40. Youngerman BE, Oh JY, Anbarasan D, Billakota S, Casadei $\mathrm{CH}$, Corrigan EK, et al: Laser ablation is effective for temporal lobe epilepsy with and without mesial temporal sclerosis if hippocampal seizure onsets are localized by stereoelectroencephalography. Epilepsia 59:595-606, 2018

41. Zack MM, Kobau R: National and state estimates of the numbers of adults and children with active epilepsy-United States, 2015. MMWR Morb Mortal Wkly Rep 66:821-825, 2017

\section{Disclosures}

Drs. Ball and Neimat report being consultants to Monteris.

\section{Author Contributions}

Conception and design: Sharma. Acquisition of data: Wang. Analysis and interpretation of data: Sharma. Drafting the article: Sharma, Ball. Critically revising the article: all authors. Reviewed submitted version of manuscript: Neimat, Sharma, Ball, Alhourani, Ugiliweneza, Wang. Statistical analysis: Ugiliweneza, Wang. Administrative/technical/material support: Neimat, Boakye. Study supervision: Neimat, Boakye.

\section{Supplemental Information Online-Only Content}

Supplemental material is available online. Supplementary Table 1. https://thejns.org/doi/suppl/10.3171/ 2020.1.FOCUS19935.

\section{Correspondence}

Joseph S. Neimat: University of Louisville, KY. joseph.neimat@ louisville.edu. 